\title{
Microscale Magneto-Elastic Composite Swimmers at the Air-Water and Water-Solid Interfaces Under a Uniaxial Field
}

\author{
M.T. Bryan, ${ }^{1, *}$ J. Garcia-Torres, ${ }^{2,3,4}$ E.L. Martin, ${ }^{1}$ J.K. Hamilton, ${ }^{1}$ C. Calero, ${ }^{2,4,5}$ P.G. Petrov, ${ }^{1}$ \\ C.P. Winlove, ${ }^{1}$ I. Pagonabarraga, ${ }^{2,4,5}$ P. Tierno, ${ }^{2,4,5}$ F. Sagués,${ }^{3,4}$ and F.Y. Ogrin ${ }^{1}$ \\ ${ }^{1}$ College of Engineering, Mathematics and Physical Sciences, University of Exeter, Exeter, UK \\ ${ }^{2}$ Condensed Matter Physics Department, Faculty of Physics, Universitat de Barcelona, Spain \\ ${ }^{3}$ Materials Science and Physical Chemistry Department, Faculty of Chemistry, Universitat de Barcelona, Spain \\ ${ }^{4}$ Institut de Nanociència i Nanotecnologia, IN ${ }^{2}$ UB, Universitat de Barcelona, Barcelona, Spain \\ ${ }^{5}$ Universitat de Barcelona Institute of Complex Systems (UBICS), Barcelona, Spain
}

(Received 24 August 2018; revised manuscript received 12 February 2019; published 8 April 2019)

\begin{abstract}
Self-propulsion of magneto-elastic composite microswimmers is demonstrated under a uniaxial field at both the air-water and the water-substrate interfaces. The microswimmers are made of elastically linked magnetically hard Co-Ni-P and soft Co ferromagnets, fabricated using standard photolithography and electrodeposition. Swimming speed and direction are dependent on the field frequency and amplitude, reaching a maximum of $95.1 \mu \mathrm{m} / \mathrm{s}$ on the substrate surface. Fastest motion occurs at low frequencies via a spinning (air-water interface) or tumbling (water-substrate interface) mode that induces transient inertial motion. Higher frequencies result in low Reynolds number propagation at both interfaces via a rocking mode. Therefore, the same microswimmer can be operated as either a high or a low Reynolds number swimmer. Swimmer pairs agglomerate to form a faster superstructure that propels via spinning and rocking modes analogous to those seen in isolated swimmers. Microswimmer propulsion is driven by a combination of dipolar interactions between the Co and Co-Ni-P magnets and rotational torque due to the applied field, combined with elastic deformation and hydrodynamic interactions between different parts of the swimmer, in agreement with previous models.
\end{abstract}

DOI: 10.1103/PhysRevApplied.11.044019

\section{INTRODUCTION}

Magnetic microscale self-propelling robots have huge potential for environmental, medical, and biotechnological applications [1] since they can be targeted toward specific areas with wireless control of direction and generate actuation at length scales approaching the cellular level. Three distinct strategies have been proposed for generating artificial self-propulsion: rotation of a helical structure [2-6], dynamic interactions within a colloidal swarm [7-10], and magnetically induced elastic flexure [11-13]. These strategies mimic solutions found in nature, corresponding to rotation of single flagella [14], swarming bacteria [15], and the coordinated beating of multiple flagella [16] or modulation of euglenid body shape [17]. Helical swimmers, fabricated via strain-engineering or threedimensional lithography, propel themselves in a rotating field through a corkscrew-like mechanism. While several applications have been demonstrated, including cargo delivery $[5,6]$, the inherently three-dimensional structure

\footnotetext{
*matthew.bryan@rhul.ac.uk
}

of a helix means that large-scale production remains a challenge [18]. Magnetic swarm swimmers utilize competition between a uniform drive field and spatially varying interactions between individual particles in a colloidal ensemble to produce collective motion as a group, even though isolated members of the swarm are unable to selfpropel. Motion due to emergent behavior is important for fundamental science, but may not be suitable for medical or microfluidic applications where shear forces from fluid flow could disrupt the balance of the complex interactions, such as in the body [19]. Magneto-elastic composite swimmers encapsulate magnetic particles within an elastic material to create a structure that deforms under a magnetic field, producing motion via a change in shape during the stroke cycle. More stable than the swarm swimmers and more compatible with standard fabrication methods than helical swimmers, magneto-elastic composite swimmers may provide the most feasible solution to generating self-propulsion at the production scales needed by applications.

Swimmer hydrodynamic behavior is characterized by the Reynolds number ( $\mathrm{Re})$, the ratio of the inertial and viscous forces. The Reynolds number is calculated from the speed $(u)$ and length $(L)$ of the swimmer in a fluid of 
kinematic viscosity $(v)$

$$
\operatorname{Re}=\frac{u L}{v} .
$$

When viscous forces dominate ( $\operatorname{Re} \ll 1$, the low Reynolds number regime), momentum is negligible, with propulsion determined by the forces acting on the swimmer at each instant of the swimming cycle rather than how fast the forces are applied. Consequently, symmetry in the cycle of strokes generating propulsion (reciprocal motion) results in equal motion in the forward and backward directions, and therefore, no overall displacement. Microscale swimmers moving in biologically relevant fluids (e.g., water, $v=1 \times 10^{-6} \mathrm{~m}^{2} \mathrm{~s}^{-1}$ ) typically translate within the low Reynolds number regime. Therefore, each of the three artificial self-propulsion strategies (helical rotation, colloidal swarming, and magneto-elastic flexure) are designed with asymmetric swimming cycles in order to generate nonreciprocal motion and produce net translation. Note, however, that since the Reynolds number is not an intrinsic property of the swimmer, a "low Reynolds number" swimmer could, in principle, operate in the high Reynolds number regime if it could be driven fast enough.

Recently, we experimentally demonstrated low Reynolds number self-propulsion of a macroscale magneto-elastic composite swimmer containing two interacting magnetic particles of different magnetic anisotropy connected by an elastic link [12]. Both the speed and direction of the two-particle swimmer could be controlled with a uniaxial field simply by varying the amplitude and frequency. Models indicted that the performance of these swimmers in bulk fluids was determined by a combination of the dipolar, elastic, and hydrodynamic interactions between the two particles and the torque due to the applied field [20] and that proximity to a boundary could alter the mode of propagation, enhancing the swimming speed [21]. In addition to generating self-propulsion, the two-particle system was also demonstrated to act as a wireless fluid pump [22] for use in disposable point-of-care diagnostic devices, which require simple mixing strategies for reagents. However, the two-particle swimmers demonstrated to date are millimeter-scale devices, too large for many microfluidic and in vivo applications. Furthermore, the mold-based fabrication method cannot easily be scaled down to the microscale dimensions needed, so new strategies are required to produce significantly smaller devices. Here, we show that magneto-elastic swimmers, consisting of elastically linked magnetically hard [Co-Ni-P] and soft (Co) particles, can be made at the microscale using standard lithographic methods, demonstrate that they can self-propel under a uniaxial field at both the air-water and the water-solid interfaces, and investigate their propulsion modes.

\section{SAMPLE FABRICATION AND MEASUREMENT}

Arrays of $68-\mu \mathrm{m}$ long magneto-elastic composite swimmers are patterned using direct-write photolithography on a glass $/ \mathrm{Cr}(5 \mathrm{~nm}) / \mathrm{Au}(100 \mathrm{~nm})$ substrate and electrodeposition of magnetic particles. Photolithography is performed in three nominally identical stages to define the shapes of the hard magnetic particles, the soft magnetic particles, and the elastic link (Fig. 1). For each photolithographic stage, three layers of Shipley S1828 photoresist are spun at (a)

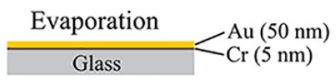

(b)

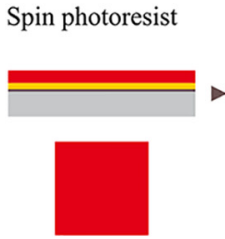

(c) Spin photoresist

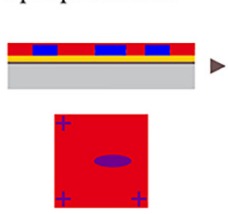

(d)

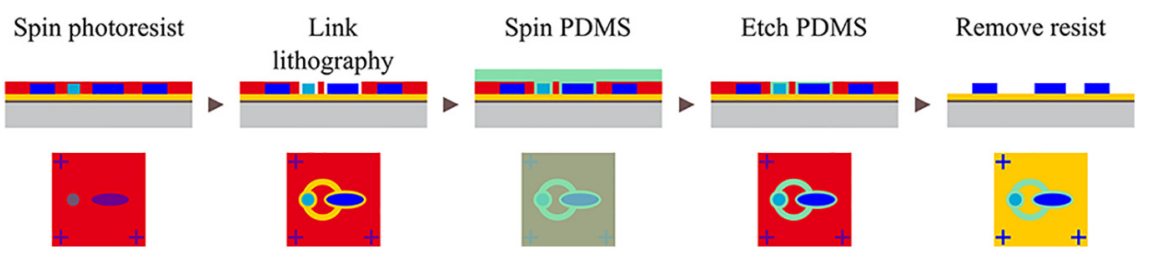

FIG. 1. Flow diagram of the fabrication process. (a) Evaporation of $\mathrm{Cr} / \mathrm{Au}$ on glass. (b) Preparation of alignment markers and the hard Co-Ni-P particles. To minimize systematic error, alignment marker lithography is performed prior to particle lithography. (c) Preparation of soft Co particles. (d) Preparation of the PDMS links. 
$2000 \mathrm{rpm}$, giving a total resist thickness of $6 \mu \mathrm{m}$. Magnetically hard Co-Ni-P particles are electroplated at $30^{\circ} \mathrm{C}$ in an aqueous solution containing $60 \mathrm{~g} / \mathrm{l}$ nickel II sulfate hexahydrate $\left(\mathrm{NiSO}_{4} \cdot 6 \mathrm{H}_{2} \mathrm{O}\right), 33 \mathrm{~g} / 1$ cobalt II sulfate heptahydrate $\left(\mathrm{CoSO}_{4} \cdot 7 \mathrm{H}_{2} \mathrm{O}\right), 3.9 \mathrm{~g} / 1$ sodium hypophosphite monohydrate $\left(\mathrm{NaH}_{2} \mathrm{PO}_{2} \cdot \mathrm{H}_{2} \mathrm{O}\right)$, and $40 \mathrm{~g} / \mathrm{l}$ ammonium chloride $\left(\mathrm{NH}_{4} \mathrm{Cl}\right)$. Soft Co particles are electroplated at $50^{\circ} \mathrm{C}$ in an aqueous solution of $200 \mathrm{~g} / 1 \mathrm{CoSO}_{4} \cdot 7 \mathrm{H}_{2} \mathrm{O}$ and $40 \mathrm{~g} / 1$ boric acid $\left(\mathrm{H}_{3} \mathrm{BO}_{3}\right)$. Photoresist is replaced between patterning of the hard and soft particles to prevent bilayer formation [Figs. 1(b) and 1(c)]. Following patterning of the elastic link shape, polydimethylsiloxane (PDMS, mixed in a 10:1 ratio of Dow Corning Sylgard 184 base and curing agent) is spun at $6000 \mathrm{rpm}$ and cured at $100{ }^{\circ} \mathrm{C}$ on a hotplate for $35 \mathrm{~min}$. Excess PDMS is removed using an inductively coupled plasma (ICP) etch with $\mathrm{SF}_{6}(10 \mathrm{sccm})$ and $\mathrm{O}_{2}$ (2 $\mathrm{sccm}$ ) to achieve a thickness of $6 \mu \mathrm{m}$, level with the height of the photoresist [Fig. 1(d)].

Completed swimmers are transferred from the fabrication substrate to a well on either the air-water interface or the water-substrate surface. When placing the swimmer at the air-water interface, the well is covered with a coverslip to minimize interference from air currents. Motion is investigated using an optical microscope incorporating a pair of Helmholtz coils, which generate a uniaxial magnetic field parallel to the plane of the water surface. Swimming is recorded using a high-speed camera at either 480 or 1500 frames per second (fps) to capture the mechanism of propulsion, or at either $50 \mathrm{fps}$ or $75 \mathrm{fps}$ to measure swimmer speed and propagation direction over a period of up to $2 \mathrm{~min}$. Particle tracking software [23] is used to trace the motion paths of the magnetic particles (or the whole swimmer) prior to and during field application.

\section{RESULTS}

\section{A. Magnetic characterization}

Figure 2(a) shows a completed swimmer after resist lift-off in acetone, consisting of a $10-\mu \mathrm{m}$ diameter circular Co particle connected to a $30-\mu \mathrm{m}$ long, $10-\mu \mathrm{m}$ wide Co-Ni-P ellipse by a $40-\mu \mathrm{m}$ diameter, $4-\mu \mathrm{m}$ wide PDMS ring. Measurements taken from arrays of nominally identical particles [Fig. 2(b)] show coercive fields of 12.7 and $55.1 \mathrm{mT}$ for the Co and Co-Ni-P particles, respectively. This demonstrates that in the swimmer, the Co particle magnetization may be reversed without significantly affecting the magnetization of the Co-Ni-P. Note, however, that these measurements are taken on pinned particles and that rotation of freely moving swimmers during alignment with the field will generate field components transverse to the easy axis of the particles. Since even small transverse fields can dramatically change domain structure and reversal field $[24,25]$, switching of the Co particle in swimmers may occur at lower fields.
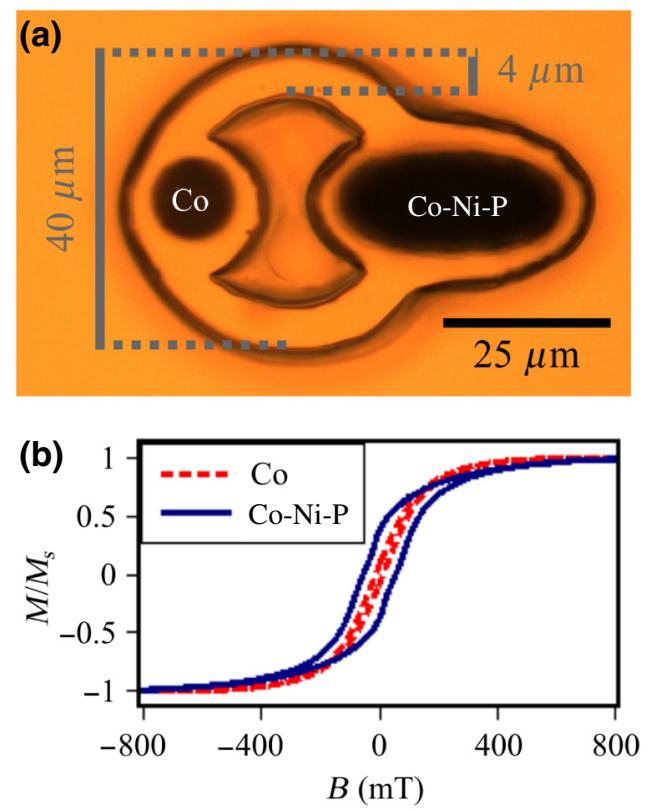

FIG. 2. (a) Optical microscopy image of a two-particle ferromagnetic swimmer with soft $\mathrm{Co}$ and hard Co-Ni-P particles labeled (body length of $68 \mu \mathrm{m}$ ). Swimmer orientation defined by the rotation of the vector from the Co particle to the Co-Ni-P particle from the field axis. (b) Vibrating sample magnetometer hysteresis loops of arrays of $\mathrm{Co}$ and $\mathrm{Co}-\mathrm{Ni}-\mathrm{P}$ particles.

\section{B. Demonstration of self-propulsion at the air-water interface}

Magnetically induced self-propulsion is apparent at the air-water interface under application of an oscillating uniaxial field, characterized by a distinct change in propagation direction from general drift seen before field application (Video 1). Figure 3(a) shows the frequency dependence of the swimming speed at field amplitudes of 3.5, 7.0, and $14.0 \mathrm{mT}$. All three amplitudes produce similar trends with peaks in swimming speed occurring around 15 and $25 \mathrm{~Hz}$, with an additional peak at $70 \mathrm{~Hz}$ in the $7.0 \mathrm{mT}$ data. Similar multipeak behavior is seen in modeling and in experimental macroscale two-particle swimmers and is shown to be due to competing torques generated by aligning the applied field perpendicular to the Earth's magnetic field (as was the case in the present experiment) [12]. The similarities between the macroscale and microscale swimmers indicate the scalability of the design concept of the two-particle swimmers.

Random drift in the absence of an applied field, caused by perturbations in the environment such as air currents, water currents, or ambient vibrations, can be confused with self-propulsion at the air-water interface. Indeed, due to the lack of damping at the air-water interface, we observe that in the absence of the external field, swimmers randomly move with an average speed of approximately $8 \mu \mathrm{m} / \mathrm{s}$. Figure 3(a) shows measurements of the swimmer speed at 


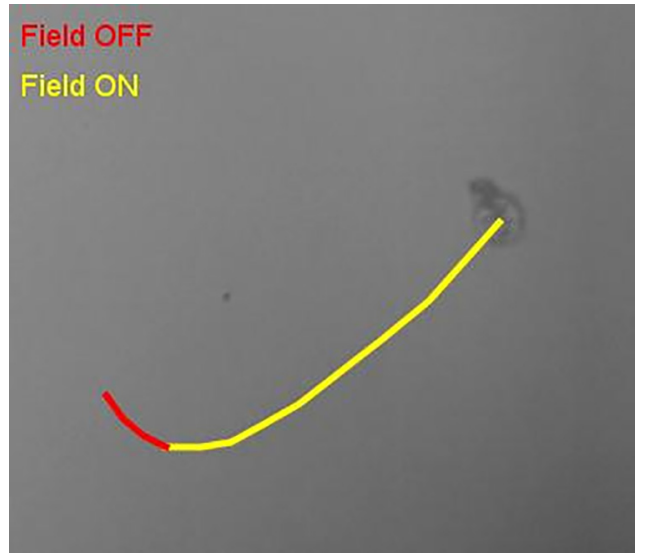

VIDEO 1. Real-time video of two-particle ferromagnetic microswimmer propagation at the air-water interface under a $3.5 \mathrm{mT}, 30 \mathrm{~Hz}$ uniaxial field. Red and yellow lines overlay the video to track motion before and during field application, respectively, plotting the swimmer position every $4 \mathrm{~s}$ (with linear interpolation between points). The motion of the swimmer before the field is applied (red track) is due to drifts experienced on the air-water interface. Note that after the application of the field, both the direction of motion and the speed change.

different frequencies and amplitudes of the applied field. Since drift in the absence of any applied field is an artifact of the experimental system rather than an intrinsic property of the swimmer, it is not possible to determine whether self-propulsion occurs in swimmers traveling slower than the drift speed. Therefore, where field conditions produce motion below $8 \mu \mathrm{m} / \mathrm{s}$ [data in the grayed area of Fig. 3(a)], the experimental data will be excluded from further analysis of self-propelled swimmer motion, directionality, and orientation. Propagation velocity varies greatly with the applied field frequency. Indeed, at 20, 40, and $50 \mathrm{~Hz}$, negligible self-propulsion occurs for all the applied field amplitudes. However, swimmer propagation is much faster than the drift speed for field frequencies of 15 and $25 \mathrm{~Hz}$ at all applied field amplitudes, with a third peak occurring in the $7.0 \mathrm{mT}$ data at $70 \mathrm{~Hz}$. This trend of the data, together with directional changes observed immediately after the field application (Video 1), lead us to conclude that the motion observed at the peak frequencies is due to controlled self-propulsion rather than random drift.

In addition to random drift, free-floating swimmers are extremely sensitive to systematic drift due to magnetic field gradients, so it is necessary to establish that the observed motion is not an artifact of the experimental system. Although the system is designed to generate a constant field across the imaging area using a Helmholtz coil configuration, it is feasible that the coils are not ideally balanced or are not positioned perfectly symmetrically about the imaging area, producing field gradients that move the swimmer via magnetic attraction rather than a selfpropulsion mechanism. In this case, the swimmer would

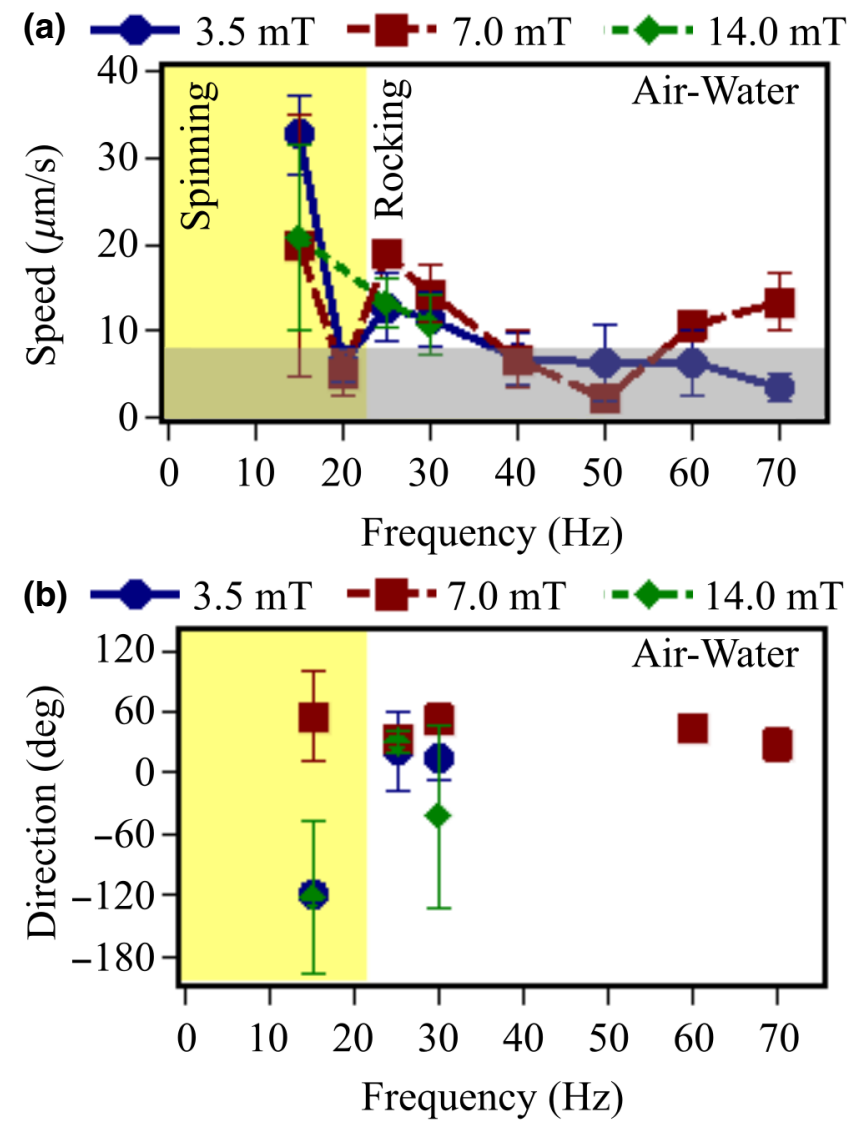

FIG. 3. Field frequency dependence of (a) swimming speed and (b) propagation direction (with respect to the field axis) of the two-particle ferromagnetic swimmer at the air-water interface under field amplitudes of 3.5, 7.0, and $14.0 \mathrm{mT}$. Data points represent the average of 24 measurements taken regularly over a 2 min period (error bars represent the standard deviation). The grayed area in (a) indicates data below the average drift speed. Directional data from these points in (b) have been neglected.

always be attracted toward the strongest or closest coil, moving in the same direction regardless of the applied frequency or field amplitude. Therefore, one way of excluding systematic drift as a cause of swimmer propagation is to drive a single swimmer in different directions, changing only the field frequency and amplitude rather than rotating the coil axis. In macroscale two-particle swimmers, the angle of trajectory is changed by up to $115^{\circ}$ by varying the frequency alone. Additionally, propagation could be directed along apparently arbitrary paths when both frequency and amplitude are varied simultaneously [12]. Low Reynolds number numerical modeling of the swimmers also reproduces these features [12], demonstrating that such behavior is not an experimental artifact. While the field amplitude determines the torque applied to the swimmer, the frequency controls the duration that the torque is applied, so both field parameters influence the amount of rotation that the swimmer undergoes in a given time 
frame. Additionally, since the soft particle reverses magnetization above a threshold field, both the field amplitude and frequency affect the timing of when dipolar forces (and consequently, elastic and hydrodynamic forces) within the swimmer change. Since the torque and magnetic interaction between the soft and hard particles are the drivers of swimming, the synchronization between them determines the trajectory of travel, enabling two-particle swimmers to be directed by the frequency and amplitude of a field applied along a single axis.

Consistent with the results of the macroscale swimmer and the numerical modeling [12,20], the microscale swimmer propagation varies with both field amplitude and frequency [Fig. 3(b), which excludes data likely to be dominated by random drift]. Notably, propagation is not directed toward either coil $\left(0^{\circ}, 180^{\circ}\right)$, as may be expected from the presence of a field gradient along the axis of the magnetic coils. Indeed, $\chi^{2}$ tests indicate that the probability of the swimmer experiencing systematic drift (movement toward any constant direction, regardless of field parameters) is insignificantly small $(<0.1 \%)$. This indicates that any magnetic gradients around the imaging area are negligible and that the observed motion is due to controlled self-propulsion rather than magnetic attraction.

\section{Propagation modes at the air-water interface}

To establish the mechanics of swimming on the airwater interface, motion is observed using a high-speed camera, taking images at $1500 \mathrm{fps}$. Spinning and rocking modes of propagation are observed depending on the frequency of the applied field. At low frequencies $(\leq 20 \mathrm{~Hz})$, motion is characterized by a spinning mode, in which the swimmer performs half rotations to synchronously align with the applied field [Fig. 4(a) and Video 2]. Spinning appears to favor clockwise rotation, with counterclockwise rotations only occurring occasionally under 3.5 -mT fields, but never occurring at higher fields. This indicates that the swimmer actuation contains at least one mechanism that breaks rotational symmetry as both rotation chiralities should be equally probable. One possible mechanism is that the magnetic anisotropy axis of the Co particle is misaligned with that of the Co-Ni-P particle. While the particles would exert equal torques after alignment with the field, reversal of the field would cause the magnetization of both particles to cant away from their magnetic easy axes, with greater canting occurring in the softer particle. This additional canting would be enough to break the torsional symmetry exerted by the particles on the overall swimmer structure and fix the rotation chirality. Alternatively, elastic relaxation of the swimmer structure, and the resultant hydrodynamic flow, may provide sufficient perturbation to bias the rotation chirality. Differential contrast, achieved by digitally subtracting consecutive video (a)

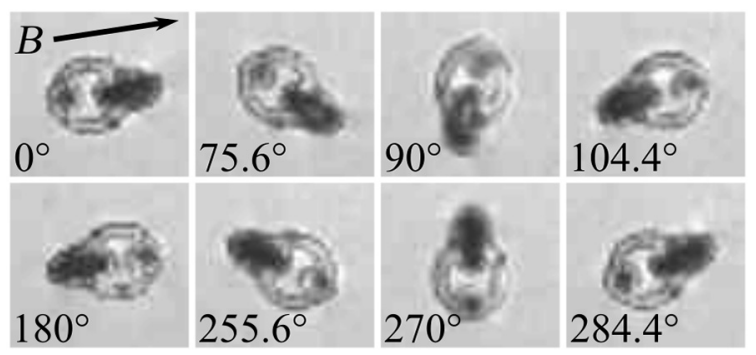

(b)

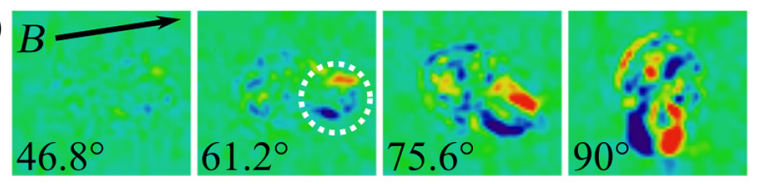

(c)

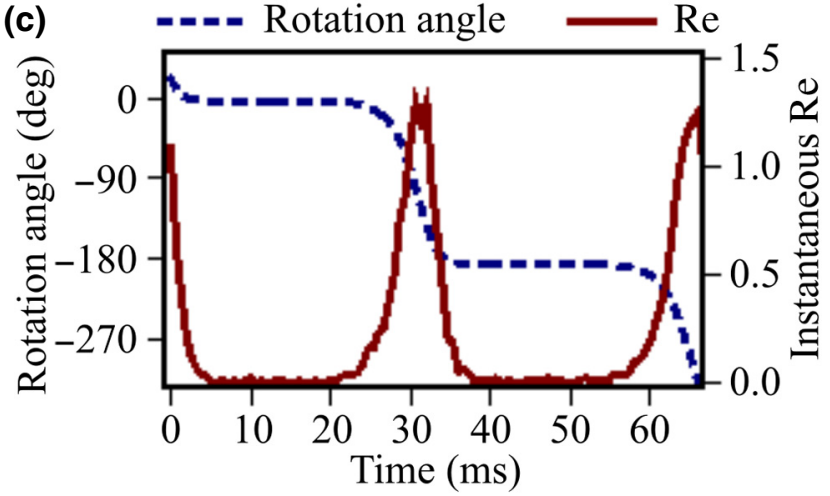

FIG. 4. (a) Representative images of spinning mode motion on the air-water interface. The phase angle within one field cycle is indicated in each image. (b) Differential images of the spinning mode, showing the change in swimmer position between the phase indicated and the previous frame (taken $0.67 \mathrm{~ms}$ earlier). Blue (red) contrast indicates regions that the swimmer has entered (left). The circled region highlights the initial distortion of the swimmer prior to rotation. (c) The time variation of the swimmer orientation and the instantaneous Reynolds number (Re) calculated from the tangential speed in the spinning mode (averaged over 10 field cycles). All data shown under a $15 \mathrm{~Hz}$, $7.0 \mathrm{mT}$ field, $B$, applied along the axis indicated by the arrows.

frames to highlight relative motion, shows a small distortion localized around the hard Co-Ni-P particle [circled region in Fig. 4(b)] prior to the rotation of the whole swimmer (Video 3). This structural deformation may also have contributed to breaking rotational symmetry by distorting the swimmer shape and producing hydrodynamic flow to bias the rotation chirality [Figs. 5(a)-5(d)].

For propulsion to be within the low Reynolds number regime, viscous drag should dominate at all time points. If $\operatorname{Re}>1$ for a fraction of the swimming cycle, then inertial effects could contribute to propagation during that time. Evaluating Eq. (1) using the average translational speed [Fig. 3(a)] indicates that $\mathrm{Re}=0.002$ at maximum, but this calculation neglects the fastest part of the motion: the tangential speed during the rotation generating the propulsion. Therefore, the tangential speed, $u$, is calculated from 


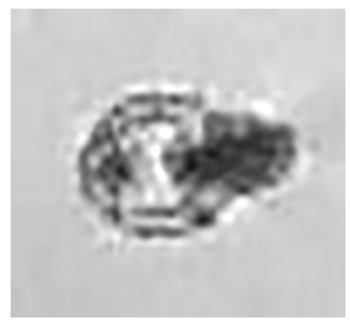

VIDEO 2. Slow-motion video of two-particle ferromagnetic microswimmer at the air-water interface moving in a spinning mode under a $15 \mathrm{~Hz}, 7.0 \mathrm{mT}$ uniaxial field. Recorded at $1500 \mathrm{fps}$, playback at $5 \mathrm{fps}$. Frames from this video are reproduced in Fig. 4(a).

the angular velocity, $\omega$, at each instant of the field cycle, treating the swimmer length $(L)$ as the diameter of rotation

$$
u=\frac{\omega L}{2} .
$$

Figure 4(c) shows the variation of the swimmer orientation and the instantaneous Reynolds number calculated from Eqs. (1) and (2) during spinning (15 Hz, $7.0 \mathrm{mT}$ field). For much of the field cycle, the swimmer is aligned with the field, so rotation is concentrated within a small time period (12.7 $\mathrm{ms}$ per $180^{\circ}$ rotation), with angular velocity peaking at $556 \mathrm{rad} / \mathrm{s}(u=19 \mathrm{~mm} / \mathrm{s})$. Figure 4(c) indicates that significant inertial forces $(\operatorname{Re}>1)$ are present in swimmer hydrodynamics for up to $23 \%$ of the rotation duration ( $9 \%$ of the field cycle). Previous models assuming $\mathrm{Re}=0$ predicted that spinning could not induce net motion [20]. Therefore, the observed propagation may have originated from transient inertial dynamics introduced by the speed of spinning. This highlights the physical significance of calculating the Reynolds number of microswimmers from the instantaneous speed of the motions generating propulsion rather than the time-averaged translational speed: slow translational speeds do not necessarily imply the swimmer operates in a low Reynolds number regime (through

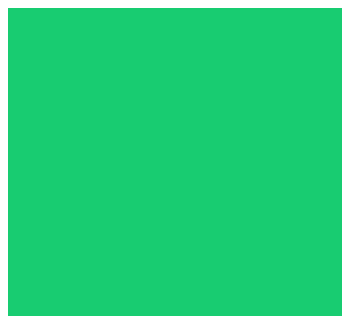

VIDEO 3. Differential video of the two-particle ferromagnetic microswimmer at the air-water interface, shown in Video 2 (spinning mode under $15 \mathrm{~Hz}, 7.0 \mathrm{mT}$ field). The differential contrast highlights the change in position between consecutive frames (taken $0.67 \mathrm{~ms}$ apart) by digitally subtracting them. A mean filter (ImageJ, radius $=1$ pixel) is used to reduce noise after the subtraction and the color scheme is chosen such that blue (red) contrast indicates regions that the swimmer has entered (left). The first frame in the sequence is blank. Frames from this video are reproduced in Fig. 4(b).

nonreciprocal action), but could be due to significant, but transient, inertial motion.

At higher frequencies $(\geq 25 \mathrm{~Hz})$, the swimmer is unable to align with the applied field before it changes direction, so it enters an asynchronous rocking mode, oscillating around a fixed orientation [Fig. 6(a) and Video 4]. Such a trapped mode has previously been identified in our analytical modeling of swimmer motion [20]. In this model, the swimmer initially rotates toward the field direction in a contracted shape [Figs. 7(a) and 7(b)]. However, the magnetization of the soft particle reverses before the rotation is complete, causing the swimmer shape to expand [Fig. 7(c)]. Nonreciprocal motion is generated because the swimmer returns to its original orientation in the expanded shape rather than the contracted shape [Fig. 7(d)]. Since rotation of the swimmer is a necessary part of the nonreciprocal action, the propagation mechanism present is fundamentally different from the "pushmepullyou" model, which mimics euglenid motion
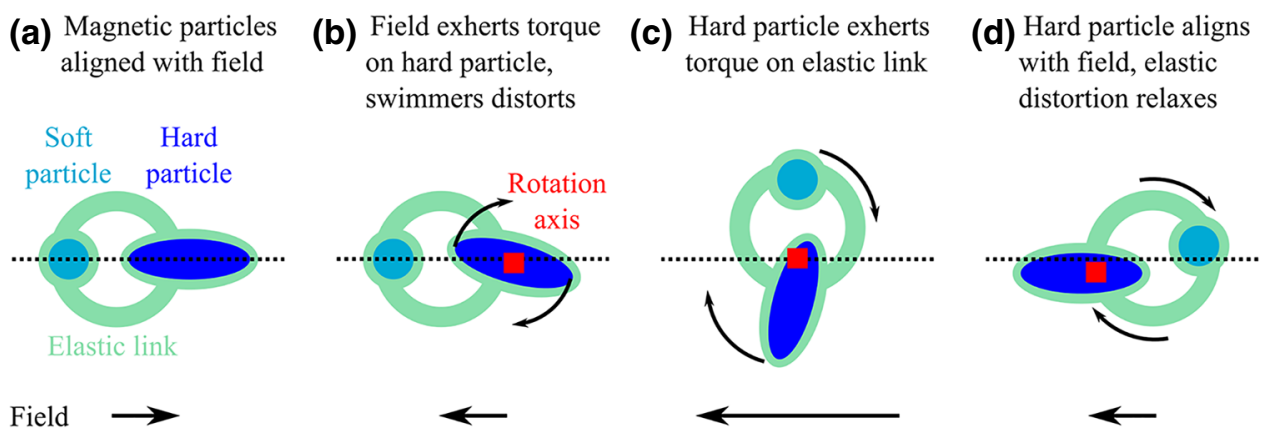

FIG. 5. (a-d) Schematic diagram of swimmer motion during the spinning: (a) magnetic particles are initially aligned with the field, (b) when the field changes direction, the torque from the field acts initially on the magnetic particles (for simplicity, we neglect the torque acting on the soft particle in this description), resulting in a localized elastic deformation and small flow around the particle, (c) rotation of the whole structure occurs, with a chirality determined by the elastic deformation and resultant flow, (d) as the particle aligns with the field, the elastic deformation relaxes and the structure unbends. 
(a)

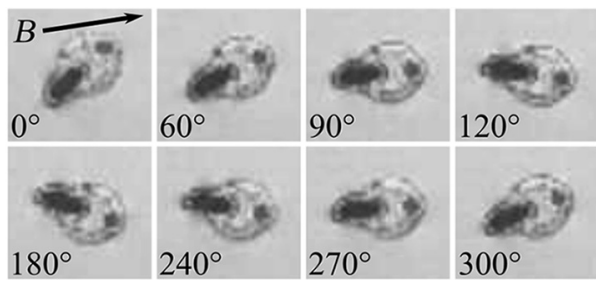

(b)
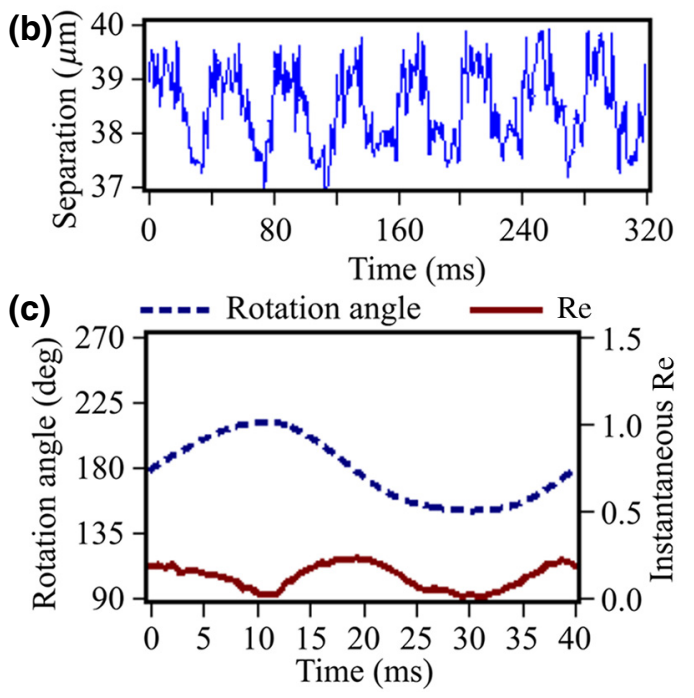

FIG. 6. (a) Representative images of rocking mode motion on the air-water interface at different phases of the field cycle $(25 \mathrm{~Hz}, 7.0 \mathrm{mT}$ field, $B$, applied along the axis indicated by the arrow). (b) Measurement of the center-to-center particle separation during rocking motion. (c) The time variation of the swimmer orientation and the instantaneous Re calculated from the tangential speed in the rocking mode (averaged over eight field cycles).

via asynchronous oscillations in the body length and the volume of the head and tail [26]. Therefore, by demonstrating that propagation can arise from a combination of rotation and body length, our magnetic swimmer may

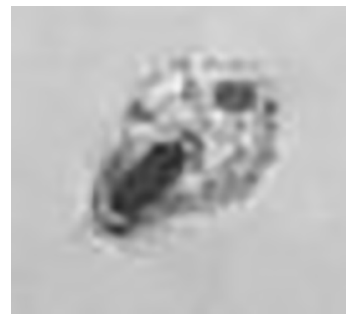

VIDEO 4. Slow-motion video of two-particle ferromagnetic microswimmer at the air-water interface moving in a rocking mode under a $25 \mathrm{~Hz}, 7.0 \mathrm{mT}$ uniaxial field. Recorded at $1500 \mathrm{fps}$, playback at $5 \mathrm{fps}$. Frames from this video are reproduced in Fig. 6(a).

provide fresh insight into possible propagation modes in these biological swimmers.

Experimentally, the lowest field amplitude applied during rocking simply perturbs the swimmer, causing the average orientation to be determined by the initial (prefield) orientation and producing a submode in which the swimmer rocks around an angle almost perpendicular to the propagation direction [Figs. 8(a) and 8(b)]. Higher fields are strong enough to align the swimmer about the field axis. Since this results in small torques, the swimmer is unable to return to the initial orientation during the reverse cycle, so the swimmer becomes trapped in a submode rocking about the field axis. Generally, the swimmer orientation is biased toward the forward field direction $\left(0^{\circ}\right)$ [Fig. 8(a)], possibly reflecting the initial torques acting on the swimmer as the field is turned on. However, rocking occasionally occurs with the swimmer oriented along the reverse field direction $\left( \pm 180^{\circ}\right)$, such as in Fig. 6(a), if the initial alignment is within $20^{\circ}$ of the reverse field direction. Rocking submodes with orientations parallel and perpendicular to the propagation direction were predicted (a) Magnetic particles aligned and attract

(b) Field exherts torque, swimmer contracted

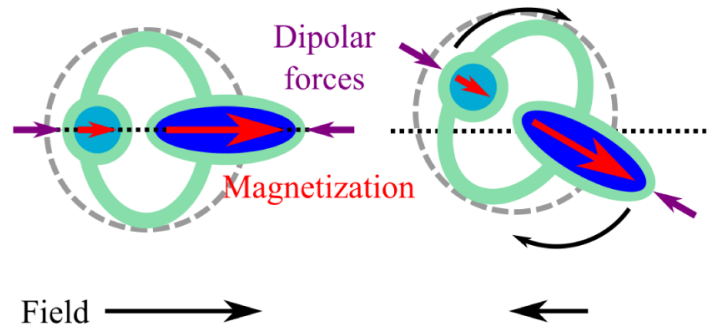

(c) Soft particle reverses, particles repel

(d) Field exherts torque, swimmer expanded
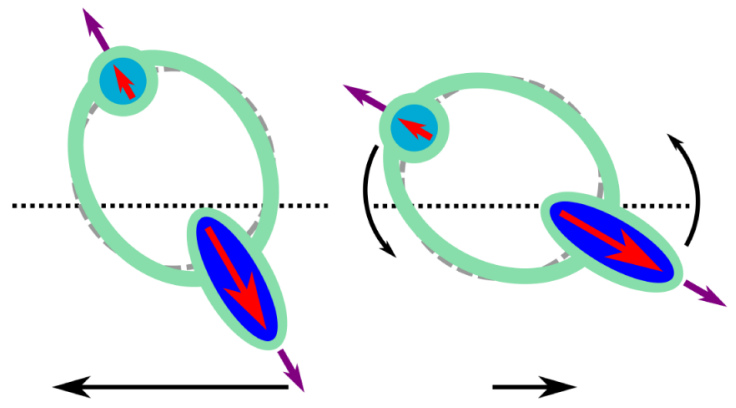

FIG. 7. (a-d) Schematic diagram of swimmer motion during the rocking mode: (a) magnetic particles are aligned with each other, causing a contraction of the swimmer (the dashed circle represents the unstrained link shape), (b) when the field changes direction, the swimmer initially rotates in the contracted state, (c) the magnetization of the soft particle reverses before the swimmer has aligned with the field, causing the swimmer to expand, (d) when the field returns to the original direction, the swimmer rotates back in the expanded state. Subsequently, the soft particle reverses again to align with the field. 

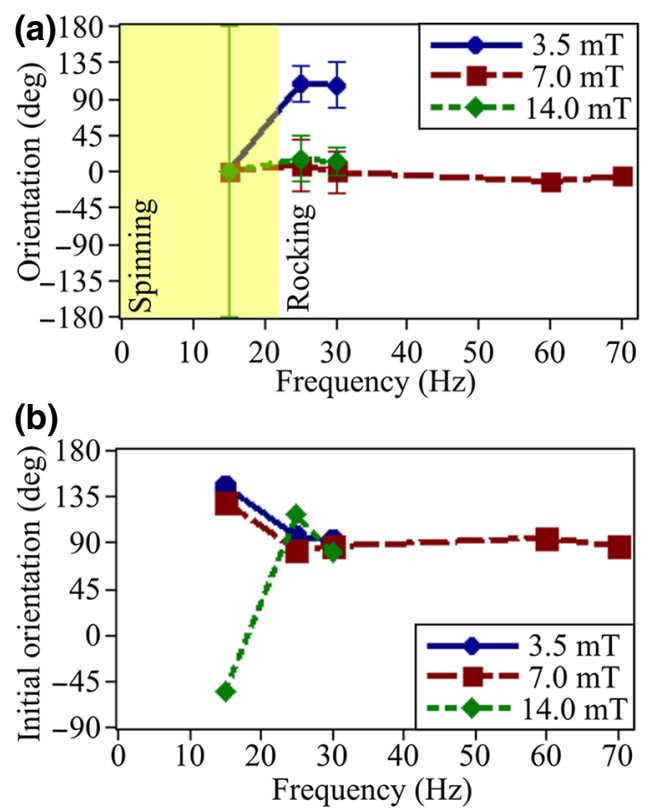

FIG. 8. (a) Swimmer orientation during propagation. Data points show the average orientation measured during speed and propagation direction measurements (shown in Fig. 3). Error bars indicate the range of angles the swimmer passes through (measured separately using $1500 \mathrm{fps}$ images). Note that in the spinning mode, the swimmer orientation rotates through $360^{\circ}$. (b) Initial orientation of the swimmer, measured immediately before the fields indicated are applied. No field is applied in (b). The field conditions shown correspond to the matching data points in (a). Orientations at $90^{\circ}$ correspond to alignment with the Earth's magnetic field.

by our previous modeling and shown to be selected by the dimensionless magnetic anisotropy of the hard and soft particles [20]. The dimensionless magnetic anisotropy describes the strength of the magnetic anisotropy energy relative to the Zeeman energy. For the hard particle, the dimensionless magnetic anisotropy is defined as

$$
\kappa_{\mathrm{hard}}=\frac{K_{\mathrm{hard}}}{K_{\mathrm{hard}}+\mu_{0} M_{\mathrm{hard}} H},
$$

where $K_{\text {hard }}$ is the uniaxial magnetic anisotropy constant of the hard particle, $\mu_{0}$ is the permeability of free space, $M_{\text {hard }}$ is the hard particle magnetization, and $H$ is the applied field strength (the soft particle dimensionless magnetic anisotropy, $\kappa_{\text {soft }}$, has a similar form). Regarding the internal magnetic response of the particles, this equation shows that increasing the field is equivalent to inducing a softer magnetic anisotropy under a constant field. Modeling with an ideally soft particle $\left(\kappa_{\text {soft }}=0\right)$ predicts that rocking will occur perpendicular to the propagation direction when the hard particle has an ideally strong dimensionless magnetic anisotropy $\left(\kappa_{\text {hard }}=1\right)$, but near parallel to the propagation at intermediate dimensionless magnetic anisotropy $\left(\kappa_{\text {hard }}=0.5\right)$ [20]. This prediction is consistent with the experimental results, where perpendicular rocking occurs at low fields (higher $\kappa_{\text {hard }}$ ) and parallel rocking is seen at higher fields (lower $\kappa_{\text {hard }}$ ).

Tracking the positions of the individual $\mathrm{Co}$ and $\mathrm{Co}-\mathrm{Ni}-\mathrm{P}$ particles during motion [23] provides a measurement of the axial deformation. Figure 6(b) shows a trace of the particle separation over eight field cycles, demonstrating a bistable oscillation between separations of 38 and $39 \mu \mathrm{m}$ with the period matching that of the applied field $(40 \mathrm{~ms})$. Despite the oscillation in the particle separation, the orientation of the swimmer varies smoothly and motion is viscosity dominated $(\mathrm{Re} \ll 1)$ throughout the field cycle [Fig. 6(c)]. Together with the consistent field dependence of the rocking submodes, this demonstrates that the propagation mechanism of the experimental microswimmer in the rocking mode is well described by our previous low Reynolds number models [12,13,20,21].

\section{Motion at the water-substrate surface}

In many proposed applications, swimmers do not float at the air-water interface, but rather propel in bulk fluids while interacting with solid surfaces such as a blood vessel wall or the sides of microfluidic channels. This changes the hydrodynamics affecting the swimmer: surface tension, present at the air-water interface, does not exist at water-solid surfaces and swimmers are less susceptible to perturbations such as the Earth's magnetic field and drift. To understand how this affects propulsion, propagation is measured for swimmers placed at the interface between water and a glass substrate [Fig. 9(a)]. Unlike at the air-water interface, the speed of the swimmer increases almost proportionally with the frequency up to a critical frequency, beyond which it decreases rapidly with increasing frequency. Below the critical frequency, near identical behavior is observed over a range of field amplitudes $(\geq 4.2 \mathrm{mT}$ ), except that stronger fields produce progressively larger critical frequencies (and therefore, maximum velocities).

As is the case at the air-water interface, the swimmer aligns synchronously with the field below the critical frequency [Fig. 9(b) and Video 5], but enters an asynchronous mode at higher frequencies (Fig. 10 and Video 6). The asynchronous modes at the air-water and water-substrate interfaces are similar [Figs. 6(a) and 10], characterized by the rocking motion described previously. However, the synchronous mode at the water-substrate interface is not a simple spinning mode, but rather a tumbling mode characterized by an out-of-plane rotation superimposed on an in-plane rotation [Fig. 9(b) and Video 5]. Tumbling may be caused by inertial hydrodynamics reflecting from the substrate, as the transient out-of-plane component of tumbling emerges and dissipates during the swimmer orientations when Re $>1$ [Figs. 9(b) and 9(c)]. Noting that the spinning mode results from a tumbling mode in which out-of-plane 


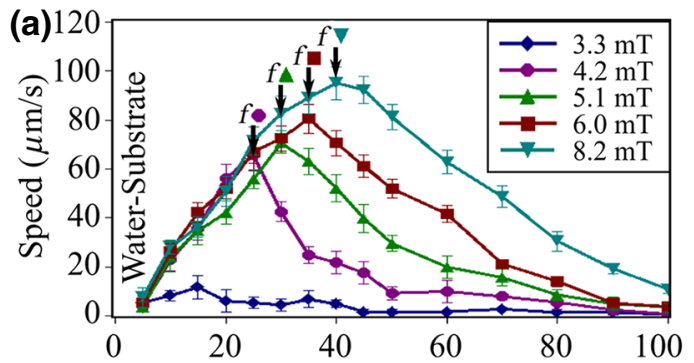

(b)

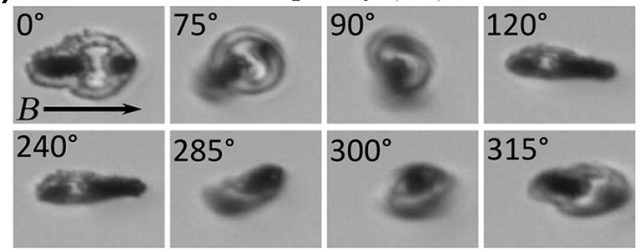

(c)

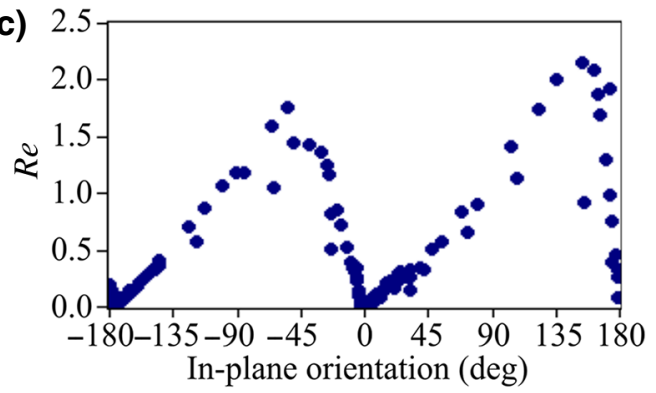

FIG. 9. (a) Frequency dependence of the swimmer speed close to the water-substrate interface. Data average of five measurements, error bars show standard deviation. Arrows denoted " $\mathrm{f}$ " mark the critical frequency above which the data indicates switches from the synchronous to the asynchronous mode. (b) Images of swimmer motion in the synchronous mode on the water-substrate interface $(20 \mathrm{~Hz}, 5.1 \mathrm{mT}$ field). The angles indicate the phase of each image within one field cycle. The arrow shows the applied field axis. (c) Relationship between the instantaneous Re calculated from the tangential speed of in-plane rotation and the azimuthal swimmer orientation (data taken over nine field cycles).

motion is suppressed, we suggest that surface tension at the air-water interface is responsible for the difference in synchronous modes seen at the two interfaces. During the tumbling mode, the swimmer moves $2.5 \pm 0.2 \mu \mathrm{m}$ per field

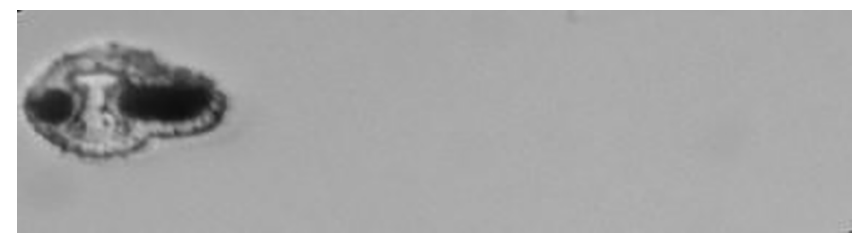

VIDEO 5. Video showing motion of the two-particle ferromagnetic microswimmer at the water-substrate surface in the synchronous tumbling mode under a $20 \mathrm{~Hz}, 5.1 \mathrm{mT}$ field. Recorded at $480 \mathrm{fps}$, playback at $2 \mathrm{fps}$. Frames from this video are reproduced in Fig. 9(b).

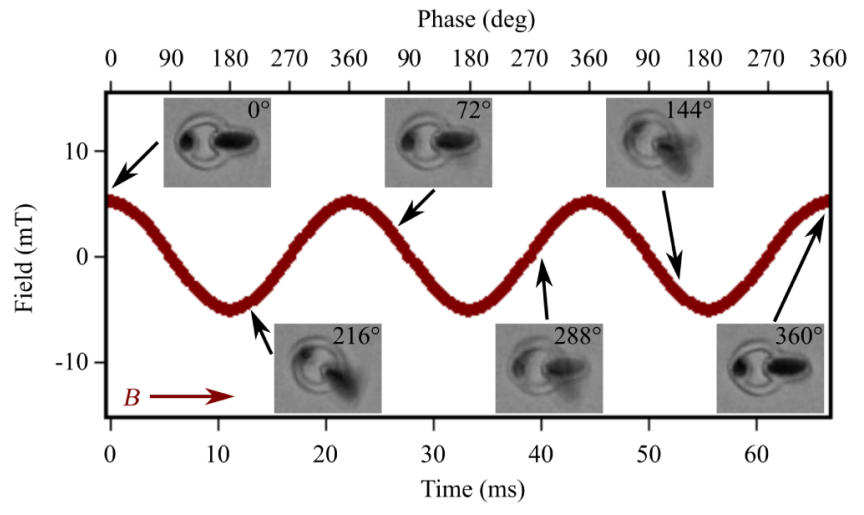

FIG. 10. Optical images of the two-particle ferromagnetic swimmer moving in the asynchronous rocking mode at the watersubstrate surface, overlaid with the trace of the $45 \mathrm{~Hz}, 5.1 \mathrm{mT}$ field applied along the axis indicated by the red arrow $(B)$. The images are taken at the time points indicated in sequential frames of the 75 fps video shown in Video 6, and the angles indicate the phase of the field cycle in which each image is taken. The rocking mode can be resolved when motion is viewed over several cycles as a function of phase angle.

cycle, regardless of the field amplitude. Such fast motion may be due to a rolling component to the tumbling, which depends on the swimmer length rather than the amplitude of the applied field.

Fastest propagation on the water-substrate surface occurs at $95.1 \mu \mathrm{m} / \mathrm{s}$, much quicker than the maximum speed observed on the air-water interface. This demonstrates that the close proximity to the substrate surface enhances the efficiency of the propulsion, as has been predicted in analytical models [21]. In the tumbling mode, the speed of the two-particle swimmer is comparable to similarly sized helical swimmers [27] (currently the fastest swimming strategy) and considerably faster than equivalent-length single-particle magneto-elastic swimmers [11] or swarm swimmers consisting of nanorodsphere assemblies [10], colloidal chains [28], or carpets

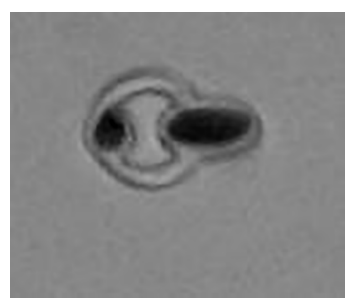

VIDEO 6. Video showing motion of the two-particle ferromagnetic microswimmer at the water-substrate surface in the asynchronous rocking mode under a $45 \mathrm{~Hz}, 5.1 \mathrm{mT}$ field. Recorded at $75 \mathrm{fps}$, playback at $2 \mathrm{fps}$. Frames from this video are reproduced in Fig. 10. 
[7]. Whereas swimming performance of the helical swimmers requires three-dimensional (3D) patterning into a corkscrew shape, the mechanism of propulsion in the twoparticle swimmers is dependent on the magnetic properties of the particles and the elastic properties of the links between them. This means that the swimming performance of two-particle swimmers is less reliant on a particular shape. Therefore, we consider the two-particle swimmer to broaden the capabilities of microswimmer technology, enabling swimmers to be fabricated using standard lithography techniques in shapes adapted for specific applications or that produce particular flow fields, while still producing fast swimming speeds.

\section{E. Cooperative motion of interacting swimmers}

In addition to self-propelling as isolated individuals, cooperative behavior emerges when multiple two-particle swimmers are placed on the air-water interface. When a swimmer is placed on the air-water interface, the interface becomes distorted due to the formation of a meniscus around the swimmer. Close proximity to another swimmer results in a hydrostatic attraction due to the meniscus, analogous to the hydrostatic interaction between beads in a recent realization of a Najafi-Golestanian microswimmer [9]. Whereas hydrostatic interactions between swimmers is largely isotropic (neglecting minor effects of swimmer shape on the meniscus), stray fields from the ferromagnetic elements preferentially align adjacent swimmers along the magnetic anisotropy axis. Therefore, pairs of swimmers on the air-water interface self-organize into a head-to-tail configuration [Fig. 11(a)i].

(a)

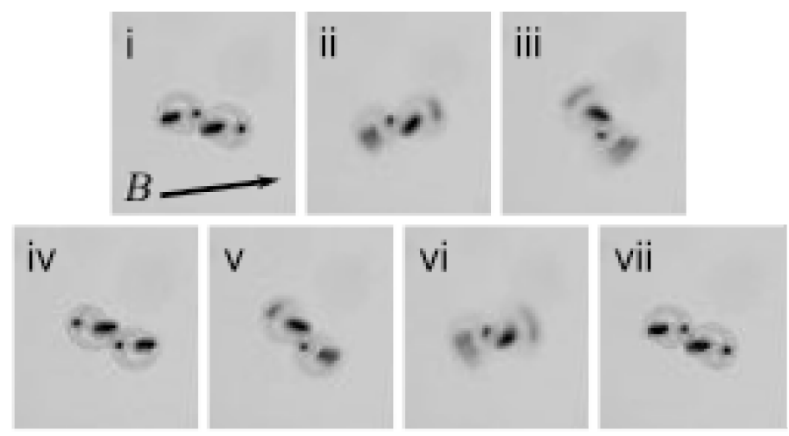

(b)

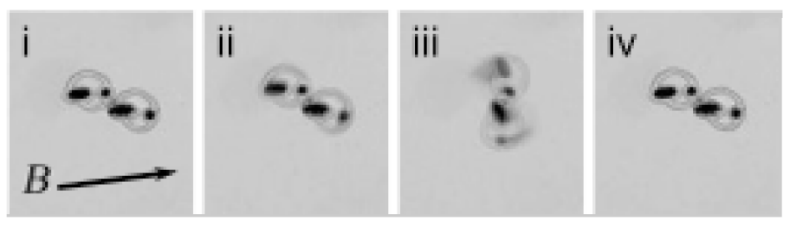

FIG. 11. (a) Spinning and (b) rocking modes exhibited by two interacting swimmers on the air-water interface under (a) $2 \mathrm{~Hz}$, $2.00 \mathrm{mT}$ and (b) $25 \mathrm{~Hz}, 3.48 \mathrm{mT}$ fields. Numerals indicate the sequence of time.
Swimmer agglomeration means that each swimmer must be considered as a building block of a superstructure rather than as an individual entity that is weakly interacting with other swimmers (as has been modeled previously [29]). Indeed, motion of the swimmer superstructure is analogous to propagation modes seen in isolated swimmers, with both spinning [Fig. 11(a)] and rocking [Fig. 11(b)] observed. Swimmer superstructures are consistently faster than isolated swimmers, with the peak speed increasing by $40 \%$ on average, indicating that hydrodynamic interactions between the swimmers act to enhance cooperative motion rather than to create destructive interference.

Looking ahead, interactions between swimmers could feasibly be controlled without fundamentally changing the swimming mechanism of individual swimmers. Since the strength of magnetic interactions between swimmers is determined by the closest separation between their magnetic elements, the width of the PDMS border surrounding the magnetic elements could be altered to tailor interactions between swimmers or to vary which elements interact. Together with the capacity to easily modify the number of magnetic elements and elastic links within the swimmer, multiparticle swimmers employ several parameters that could modify collective behavior, providing potential methods of controlling self-organization and emergent dynamics.

\section{CONCLUSIONS}

In summary, magneto-elastic composite microswimmers containing two magnetic particles of different coercivity are fabricated using widely available lithographic processes. Uniquely, the two-particle microswimmers demonstrate self-propulsion, both at the air-water interface and the water-substrate surface, under a field applied along only a single axis. At low frequencies, propagation occurs via synchronous alignment with the magnetic field in either a single-axis spinning mode (air-water interface) or multi-axis tumbling mode (water-substrate surface). Instantaneous Reynolds number calculations indicate that propagation during these modes may be assisted by transient inertial hydrodynamics generated by the speed of rotation. At higher frequencies, motion is asynchronous at both interfaces, characterized by low Reynolds number propulsion via a rocking mode where the swimmer fails to track the direction of the applied field. Therefore, under different field conditions, the same microswimmer could be propelled either through low Reynolds number mechanisms $(\operatorname{Re} \ll 1)$ or through high Reynolds number effects $(\operatorname{Re}>1)$. During motion, the swimmer shape distorts elastically due to the interaction of the magnetic particles with the applied field and with each other. Symmetry of movement is broken by interplay between this elastic deformation, the different magnetic responses of the 
particles, and the resultant hydrodynamic effects. Swimming is fastest when the swimmer is actuated close to a solid surface. This is important for many applications, since proximity to a surface is essential for practical uses of swimmers in systems such as in microfluidics or in blood vessels.

Looking beyond our specific two-particle swimmer, the methods we employ to embed magnetic particles within elastic structures could easily be adapted to produce alternative swimmer designs. Performance of magneto-elastic composite swimmers is dependent on interactions between magnetic, elastic, and hydrodynamic forces, all of which our method enables designers to control through choice of magnetic material, number and separation of magnetic particles, and link structure. Having established the principle that two-particle microswimmers can self-propel, this work paves the way for development of swimmers with arbitrary shape containing two, three, or more magnetic particles in order to produce specific swimming behavior, collective motion, or flow profiles. Furthermore, since our work on macroscale swimmers demonstrates the principle of converting swimmers into fluid pumps [22], it is feasible that the techniques developed here could enable the fabrication of wireless microscale pumps and stirrers for use in microfluidic technologies.

\section{ACKNOWLEDGMENTS}

The work was funded by the European Union's Horizon 2020 research and innovation programme Grant No. 665440. Platform Kinetics Ltd provided custom built magneto-optical equipment for observing and activating swimmers. We also acknowledge support from the EPSRC Centre for Doctoral Training in Metamaterials (Grant No. EP/L015331/1).

[1] B. J. Nelson, I. K. Kaliakatsos, and J. J. Abbott, Microrobots for minimally invasive medicine, Annu. Rev. Biomed. Eng. 12, 55 (2010).

[2] A. Barbot, D. Decanini, and G. Hwang, On-chip microfluidic multimodal swimmer toward 3D navigation, Sci. Rep. 6, 19041 (2016).

[3] W. Gao, X. Peng, A. Pei, C. R. Kane, R. Tam, C. Hennessy, and J. Wang, Bioinspired helical microswimmers based on vascular plants, Nano Lett. 14, 305 (2014).

[4] H. W. Huang, M. S. Sakar, A. J. Petruska, S. Pane, and B. J. Nelson, Soft micromachines with programmable motility and morphology, Nat. Commun. 7, 12263 (2016).

[5] F. M. Qiu, S. Fujita, R. Mhanna, L. Zhang, B. R. Simona, and B. J. Nelson, Magnetic helical microswimmers functionalized with lipoplexes for targeted gene delivery, Adv. Funct. Mater. 25, 1666 (2015).

[6] M. Medina-Sanchez, L. Schwarz, A. K. Meyer, F. Hebenstreit, and O. G. Schmidt, Cellular cargo delivery: toward assisted fertilization by sperm-carrying micromotors, Nano Lett. 16, 555 (2016).

[7] F. Martinez-Pedrero and P. Tierno, Magnetic Propulsion of Self-Assembled Colloidal Carpets: Efficient Cargo Transport via a Conveyor-Belt Effect, Phys. Rev. Appl. 3, 051003 (2015).

[8] A. Snezhko and I. S. Aranson, Magnetic manipulation of self-assembled colloidal asters, Nat. Mater. 10, 698 (2011).

[9] G. Grosjean, M. Hubert, G. Lagubeau, and N. Vandewalle, Realization of the Najafi-Golestanian microswimmer, Phys. Rev. E 94, 021101 (2016).

[10] J. Garcia-Torres, C. Calero, F. Sagués, I. Pagonabarraga, and P. Tierno, Magnetically tunable bidirectional locomotion of a self-assembled nanorod-sphere propeller, Nat. Commun. 9, 1663 (2018).

[11] I. S. M. Khalil, H. C. Dijkslag, L. Abelmann, and S. Misra, MagnetoSperm: A microrobot that navigates using weak magnetic fields, Appl. Phys. Lett. 104, 223701 (2014).

[12] J. K. Hamilton, P. G. Petrov, C. P. Winlove, A. D. Gilbert, M. T. Bryan, and F. Y. Ogrin, Magnetically controlled ferromagnetic swimmers, Sci. Rep. 7, 44142 (2017).

[13] F. Y. Ogrin, P. G. Petrov, and C. P. Winlove, Ferromagnetic Microswimmers, Phys. Rev. Lett. 100, 218102 (2008).

[14] M. Morse, A. Huang, G. L. Li, M. R. Maxey, and J. X. Tang, Molecular adsorption steers bacterial swimming at the air/water interface, Biophys. J. 105, 21 (2013).

[15] A. Yang, W. S. Tang, T. Y. Si, and J. X. Tang, Influence of physical effects on the swarming motility of Pseudomonas aeruginosa, Biophys. J. 112, 1462 (2017).

[16] K. Y. Wan, K. C. Leptos, and R. E. Goldstein, Lag, lock, sync, slip: the many 'phases' of coupled flagella, J. R. Soc. Interface 11, 20131160 (2014).

[17] M. Arroyo, L. Heltai, D. Millan, and A. DeSimone, Reverse engineering the euglenoid movement, Proc. Natl. Acad. Sci. U.S.A. 109, 17874 (2012).

[18] A. Fernandez-Pacheco, R. Streubel, O. Fruchart, R. Hertel, P. Fischer, and R. P. Cowburn, Three-dimensional nanomagnetism, Nat. Commun. 8, 15756 (2017).

[19] H. Samady, P. Eshtehardi, M. C. McDaniel, J. Suo, S. S. Dhawan, C. Maynard, L. H. Timmins, A. A. Quyyumi, and D. P. Giddens, Coronary artery wall shear stress is associated with progression and transformation of atherosclerotic plaque and arterial remodeling in patients with coronary artery disease, Circulation 124, 779 (2011).

[20] A. D. Gilbert, F. Y. Ogrin, P. G. Petrov, and C. P. Winlove, Theory of ferromagnetic microswimmers, Q. J. Mech. Appl. Math. 64, 239 (2011).

[21] M. T. Bryan, S. R. Shelley, M. J. Parish, P. G. Petrov, C. P. Winlove, A. D. Gilbert, and F. Y. Ogrin, Emergent propagation modes of ferromagnetic swimmers in constrained geometries, J. Appl. Phys. 121, 073901 (2017).

[22] J. K. Hamilton, M. T. Bryan, A. D. Gilbert, F. Y. Ogrin, and T. O. Myers, A new class of magnetically actuated pumps and valves for microfluidic applications, Sci. Rep. 8, 933 (2018).

[23] D. Brown, Tracker. (2016) http://physlets.org/tracker.

[24] M. T. Bryan, P. W. Fry, T. Schrefl, M. R. J. Gibbs, D. A. Allwood, M. Y. Im, and P. Fischer, Transverse fieldinduced nucleation pad switching modes during domain wall injection, IEEE Trans. Magn. 46, 963 (2010). 
[25] M. T. Bryan, D. Atkinson, and D. A. Allwood, Multimode switching induced by a transverse field in planar magnetic nanowires, Appl. Phys. Lett. 88, 032505 (2006).

[26] J. E. Avron, O. Kenneth, and D. H. Oaknin, Pushmepullyou: An efficient micro-swimmer, New J. Phys. 7, 234 (2005).

[27] S. Tottori, L. Zhang, F. Qiu, K. K. Krawczyk, A. Franco-Obregon, and B. J. Nelson, Magnetic helical micromachines: fabrication, controlled swimming, and cargo transport, Adv. Mater. 24, 811 (2012).

[28] F. Martinez-Pedrero, A. Ortiz-Ambriz, I. Pagonabarraga, and P. Tierno, Colloidal Microworms Propelling via a Cooperative Hydrodynamic Conveyor Belt, Phys. Rev. Lett. 115, 138301 (2015).

[29] A. D. Gilbert, F. Y. Ogrin, P. G. Petrov, and C. P. Winlove, Motion and mixing for multiple ferromagnetic microswimmers, Eur. Phys. J. E 34, 121 (2011). 